\title{
A shape-shifting nuclease unravels structured RNA
}

\author{
Katarina Meze ${ }^{1,2,3}$, Armend Axhemi ${ }^{1,3}$, Dennis R. Thomas ${ }^{1,3}$, Ahmet Doymaz $^{2,4}$ and \\ Leemor Joshua-Tor ${ }^{1,2,3 *}$
}

${ }^{1}$ W.M. Keck Structural Biology Laboratory, Howard Hughes Medical Institute, 1 Bungtown Road, Cold Spring Harbor, 11724 , USA.

${ }^{2}$ Cold Spring Harbor School of Biological Sciences

${ }^{3}$ Cold Spring Harbor Laboratory, 1 Bungtown Road, Cold Spring Harbor, NY, 11724, USA.

${ }^{4}$ Current address: Weill Cornell/Rockefeller/Sloan Kettering Tri-Institutional MD-PhD Program, New York, NY 10065, USA

*To whom correspondence should be addressed

Correspondence:

Leemor Joshua-Tor

leemor@cshl.edu 
RNA turnover pathways ensure appropriate gene expression levels by eliminating unwanted transcripts that may otherwise interfere with cellular programs. The enzyme Dis3-like protein 2 (Dis3L2) is a 3'-5' exoribonuclease that, through its RNA turnover activity, plays a critical role in human development ${ }^{1}$. Dis3L2 can independently degrade structured substrates and its targets include many coding and non-coding 3'-uridylated RNAs ${ }^{1-5}$. While the basis for Dis3L2's substrate recognition has been well-characterized ${ }^{6}$, the mechanism of structured RNA degradation by this family of enzymes is unknown. We characterized the discrete steps of the degradation cycle by determining electron cryo-microscopy structures representing snapshots along the RNA turnover pathway and measuring kinetic parameters for single-stranded (ss) and double-stranded (ds) RNA processing. We discovered a dramatic conformational change that is triggered by the dsRNA, involving repositioning of two cold shock domains by $70 \AA$. This movement exposes a trihelix-linker region, which acts as a wedge to separate the two RNA strands. Furthermore, we show that the trihelix linker is critical for dsRNA, but not ssRNA, degradation. These findings reveal the conformational plasticity of this enzyme, and detail a novel mechanism of structured RNA degradation. 
Meze et al.

RNA quality control and turnover are vital for cellular function, yet little is known about how nucleases deal with the diverse universe of structured RNAs. Dis3L2 is an RNAse II/R family 3'-5' hydrolytic exoribonuclease that plays an important role in development and differentiation ${ }^{1,7}$, cell proliferation ${ }^{8-11}$,

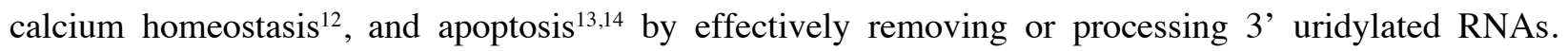
Dis3L2 targets are oligo-uridylated by the terminal uridylyl-transferases (or TUTs) ${ }^{15-17}$. The specificity towards uridylated RNAs is conferred through a network of base-specific H-bonds along the protein's extensive RNA binding surface, as demonstrated by the structure of M. musculus (Mm) Dis3L2 in complex with a $\mathrm{U}_{13} \mathrm{RNA}^{6}$.

Genetic loss of Dis3L2 causes Perlman Syndrome, a congenital overgrowth disorder that is characterized by developmental delay, renal abnormalities, neonatal mortality, and high rates of Wilms' tumors $^{1}$. The first reported physiological substrates of Dis3L2 were the uridylated precursors of let-7 microRNAs (miRNA) ${ }^{2,5}$ which play an important role in stem-cell differentiation by silencing growth and proliferation genes such as HMGA2, MYC and Ras ${ }^{18-23}$. Many other non-coding RNA targets have since been reported, including other miRNAs ${ }^{24,25}$, tRNA fragments ${ }^{17}$, snRNA $^{26}$, the intermediate of $5.8 \mathrm{~S}$ ribosomal RNA processing $7 \mathrm{~S}_{\mathrm{B}}{ }^{27}$, the long non-coding RNA $\mathrm{RMRP}^{28}$, and the $7 \mathrm{SL}$ component of the ribonucleoprotein signal recognition particle required for ER-targeted translation ${ }^{12}$. The latter is most likely responsible for the Perlman syndrome phenotype, with aberrant uridylated 7SL leading to ER calcium leakage that perturbs embryonic stem cell differentiation particularly in the renal lineage ${ }^{12}$.

Unlike a number of structurally similar homologs, Dis3L2 can degrade structured RNAs independent of external helicase activity ${ }^{1,2,4,29}$. Little is known about how Dis3L2 (or other capable RNase R/II family nucleases) independently degrades structured RNA. We determined the first structures of an RNase R/II family nuclease bound to a series of structured RNA substrates and analyzed the kinetic profiles of wildtype (WT) and mutant H. Sapiens (Hs) Dis3L2 at single nt resolution, to reveal how this nuclease achieves highly efficient degradation of structured RNA. 
Meze et al.

\section{Initial Binding of Dis3L2 to Structured Substrates}

To understand the pre-substrate binding state, we used cryo-electron microscopy (cryoEM) to determine the structure of RNA-free HsDis3L2 to $3.4 \AA$ resolution (construct Dis3L2 ${ }^{\text {D391N }}$ : residues 1-858 C-terminal truncation; catalytic mutant D391N) (see Methods, Fig. 1a, b, Extended data Fig. 1a-c). RNAfree HsDis3L2 has a vase-like conformation in which three OB domains - two cold shock domains (CSDs) and an S1 domain - encircle a funnel-like tunnel that reaches into the RNA-binding domain (RNB) and leads to the active site (Fig. 1b, Extended data Fig. 1d, e). The OB domains provide a large positively charged surface, which likely acts as a landing pad for the negatively-charged RNA (Extended data Fig. 1f). This structure is very similar to the structure of the mouse Dis3L2-ssRNA complex (M. musculus (Mm)Dis3L2- $\mathrm{U}_{13}$ ) (RMSD $1.2 \AA$ calculated over all C $\alpha$ pairs $)^{6}$. Thus, the apo-enzyme is preorganized to bind ssRNA.

To probe the initial binding of Dis3L2 to structured substrates we designed a short-hairpin RNA mimicking the base of the pre-let-7g stem, with a UUCG tetraloop for stability, and a 3' $-\mathrm{GC}(\mathrm{U})_{14}(16 \mathrm{nt})$ overhang as the uridylated tail (hairpinA-GCU ${ }_{14}$, Extended Data Fig. 2a). The resulting $3.1 \AA$ cryoEM structure of the Dis3L2 $2^{\text {D391N-hairpinA-GCU }}{ }_{14}$ complex revealed that Dis3L2 maintains the same vase conformation as observed in the RNA-free form (Fig. 1c, Extended data Fig. 2b-d). However, the doublehelical stem of the RNA was not resolved, suggesting that dsRNA is not stably engaged by the nuclease upon initial substrate association. Nonetheless, the quality of the density allowed assignment of 15 out of the 16 nucleotides of the ss-3' overhang. The RNA follows the same path as seen in the MmDis3L2- $\mathrm{U}_{13}$ structure (RMSD of $0.8 \AA$ over C4 atom pairs), and also forms numerous base-specific hydrogen bonds with the protein (Extended data Fig. 2e-i). As in the MmDis3L2-U $\mathrm{U}_{13}$ structure, seven nucleotides at the 3' end are buried in the RNB domain tunnel (Extended data Fig. 2i). This tunnel can only accommodate ssRNA, which means that as Dis3L2 degrades through the ssRNA overhang and the dsRNA is brought into contact with the nuclease, the double strand will have to unwind for degradation to continue. 


\section{3' tail shortening brings the RNA double helix into contact with the CSD and S1 domains}

To examine the structural changes occurring upon substrate processing, we shortened the 3' overhang to 12 uridines and further modified the stem to increase stability (hairpinC- $\mathrm{U}_{12}$, Extended data Fig. 3a). We obtained a $3.1 \AA$ A structure of wild-type (WT) HsDis3L2 with hairpinC-U $\mathrm{U}_{12}$ in which the double-helical stem of the RNA hairpin is clearly visible and nestled between the two CSDs and the S1 domain (Fig. 1d, Extended data Fig. 3b-d). The basal junction of the hairpin interacts with the S1 and CSD1 domains, while the apical loop interacts with CSD2 (Extended data Fig. 3e-g). At this point, when the 3' overhang is 12-nt long, the 5' end, at the ss-ds junction, moves towards a loop in CSD1 (N76-H81), suggesting that further tail shortening would lead to a steric clash between the dsRNA stem and CSD1.

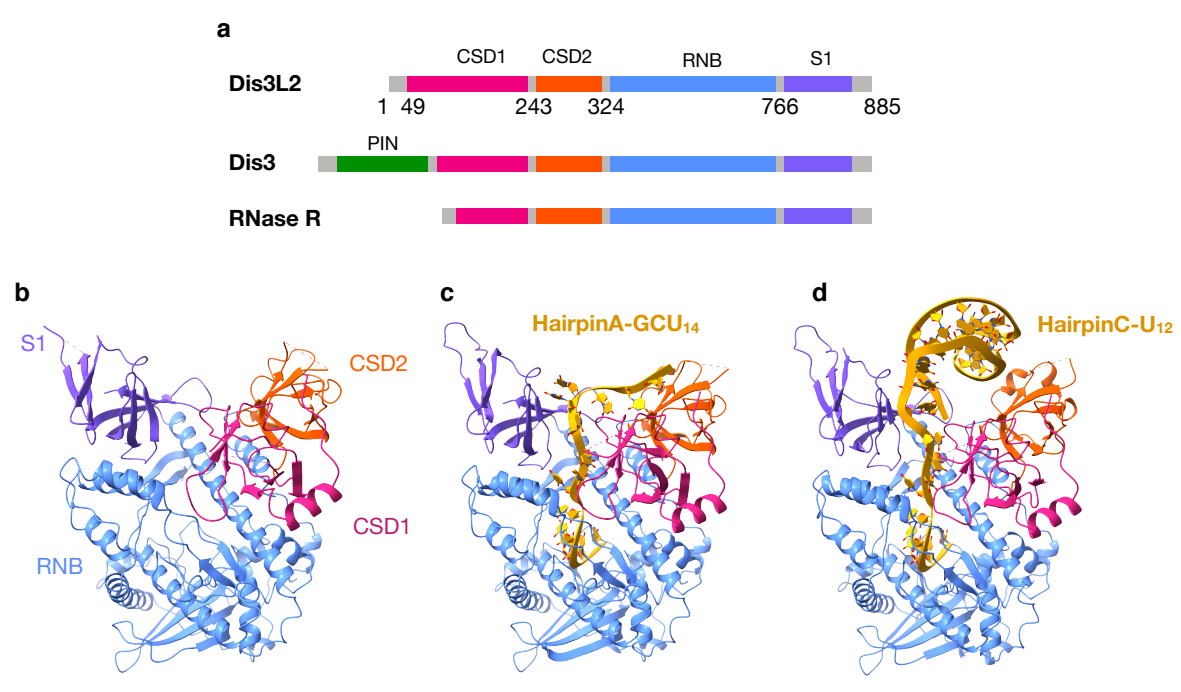

Figure 1. CryoEM structures of HsDis3L2 in complex with RNA substrates represent snapshots along the degradation pathway. a, Domain composition of Dis3L2 and homologous proteins Dis 3 and RNase R: N-terminal PIN domain (green), Cold Shock Domain (CSD1-pink, CSD2-orange), RNA-binding domain (RNB-blue), S1 domain (purple). b, RNA-free Dis3L2 $2^{\mathrm{D} 391 \mathrm{~N}}$ with domain labels. C, Dis3L2 ${ }^{\mathrm{D} 391 \mathrm{~N}}$ in complex with hairpinA-GCU ${ }_{14}$. d, WT Dis3L2 in complex with hairpinC-U $\mathrm{U}_{12}$.

\section{RNA shortening triggers a drastic conformational rearrangement of Dis3L2 prior to dsRNA unwinding}

Next, we designed a substrate with an even shorter, 7-nucleotide, 3'-overhang (hairpinD- $\mathrm{U}_{7}$ ), since this is the minimal ssRNA length needed to reach the active site from the opening to the tunnel (Extended data Fig. 2h, i, 4a). CryoEM analysis of WT HsDis3L2 in complex with hairpinD- $\mathrm{U}_{7}$ resulted in a $2.8 \AA$ A structure, 
Meze et al.

the highest resolution Dis3L2 structure reported to date (Fig. 2a, Extended Data figure 4b-d). Strikingly, it is immediately evident that the conformation of this complex is markedly different from the vase conformation observed thus far (Extended Data figure $4 \mathrm{~b}$ ). The two CSDs moved $\sim 70 \AA$ clear to the other side of the vase rim via a hinge in the linker region between CSD2 and the RNB domain (Extended Data figure 4e, SI Movie 1). This resulted in a new conformation reminiscent of a "prong" when viewed from the side (Fig. 2a). This large rearrangement is accompanied by smaller conformational changes in the S1 and RNB. The S1 domain moves such that it angles toward the double-helix where it forms new interactions with nucleotides C15 and G16 in the backbone of the double-helix, while a loop in the RNB moves by $10 \AA$ in response to the new positioning of the CSDs (Extended data Fig. $4 \mathrm{~g}, \mathrm{~h}$ ).

The two CSDs move as a block, their relative orientations unchanged. Alignment of RNA-free and hairpinD-U - -Dis3L2 structures shows that the 5' strand of the ds-RNA hairpin would clash with CSD1 (Fig 2b). Thus, it appears that upon shortening of the ss-RNA overhang, the structured portion of the RNA substrate pokes the enzyme and triggers this large rearrangement. The consequence of this movement is that it allows the structured portion of the RNA to come much closer to the RNB, while also shortening the length of the narrow tunnel to the active site by two nucleotides (Extended data Fig 5a, f). Moreover, the RNA double-helical stem is now positioned on top of the junction between a bundle of three RNB helices and a linker connecting them to the rest of the RNB domain (Fig 2a, Extended data Fig 5a, b). This junction could then act as a wedge to separate the two strands of RNA, allowing the 3' strand to enter into the narrow tunnel. Six out of seven residues in the ss-overhang are in the same position as in the hairpinA-CGU $\mathrm{CU}_{14}$ and hairpinC- $U_{12}$ structures, with five fully buried in the tunnel of the RNB. There is no change in the final approach to the active site. However, the $7^{\text {th }}$ base from the $3^{\prime}$ end, which is also the first ss-base, is no longer pointing towards the S1 domain and N663 as it is in the vase conformation (Extended data Fig 5c, d). Instead, it flips to stack underneath G16 of the ds-stem and forms a pseudo base-pair with R616 which emanates from the start of three $\alpha$-helices in the RNB domain (Extended data Fig. 5b, e). 
Meze et al.

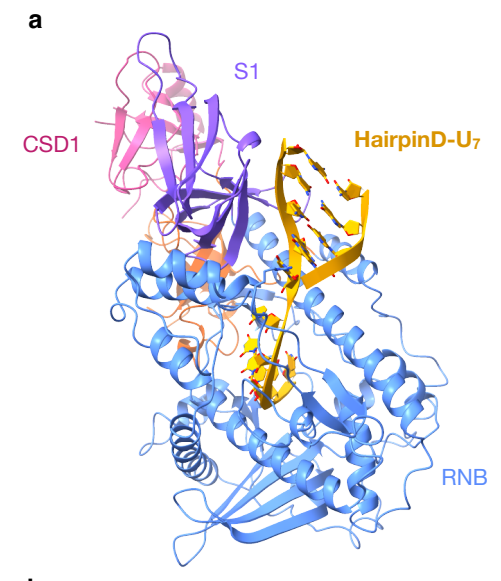

b

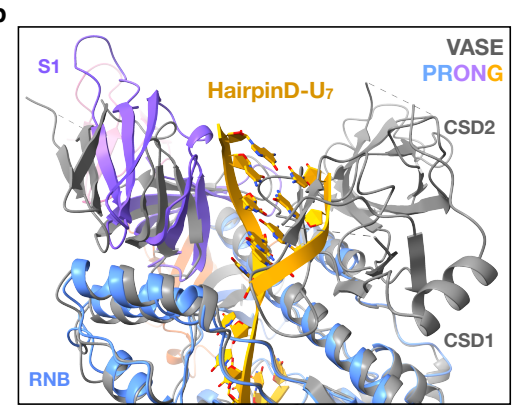

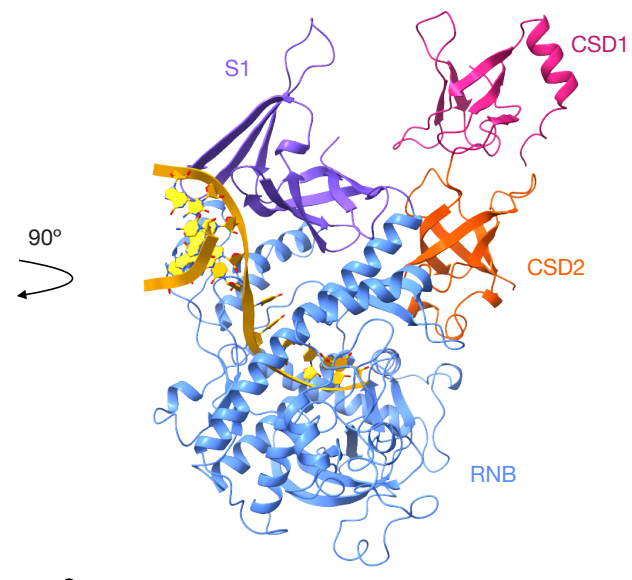

c

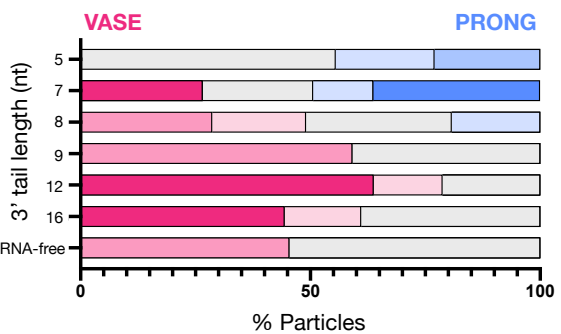

Figure 2. Dis3L2 undergoes a conformational change at $8 \mathrm{nt}$ overhang lengths. a, Two views of WT Dis3L2 in complex with hairpinD-U ${ }_{7} \mathbf{b}$, Alignment of RNA-free Dis3L2 (gray) and Dis3L2-hairpinD-U (colored domains and RNA). The CSD domains are positioned behind S1 in the prong. c, Distribution of particles after heterogeneous refinement for select datasets. X-axis: \% of particles in vase (pink) or prong (blue) conformation, y-axis: individual datasets RNA-free or hairpin RNA-bound Dis3L2, numbers denote the length of the 3' overhang. The deeper color indicates higher quality 3D-reconstructions, gray indicates particles that did not contribute to a meaningful reconstruction.

Upon close examination of our cryoEM data, we noticed that heterogeneous refinement yielded not only the high-resolution structure of the prong, but also a smaller 3D class representing the vase. Using a standardized analysis, we examined cryoEM data of Dis3L2 with a series of substrates of varying ssoverhang lengths and looked at their particle distributions between 3D classes after heterogeneous refinement (Methods, Extended data Fig. 6a). This analysis revealed the point in which this drastic conformational change occurs. The vase conformation is the only one observed in the RNA-free form and with long ss- 3'-overhangs (Fig. 2c, Extended data Fig. 6b). The prong conformation is first observed when the overhang is 8-nt long, and is the only conformation observed when the overhang length is shortened to $5 \mathrm{nt}$ (Figure 2c, Extended data Fig. 6b). This illustrates the shape-shifting nature of the enzyme to enable 
Meze et al.

the degradation of structured RNA substrates, and suggests that this dramatic conformational change is triggered by the RNA when the overhang is roughly 8-nt long.

\section{Dis3L2 degrades structured substrates with high processivity}

To quantitatively understand how the structural features described above impact Dis3L2's function, we carried out pre-steady state kinetic assays and pulse chase experiments to measure Processivity $(P)$, and elementary rate constants for RNA-binding (association: $k_{o n}$, dissociation: $k_{o f f}$ ) and degradation (forward step: $k_{f}$ ) for wildtype and various mutant Dis3L2 at single-nt resolution (Extended data Fig. 7a-g). Using a ${ }^{32} \mathrm{P}-5$ '-radiolabeled 34-nt hairpin RNA with a 7-base pair stem and a 16-nt overhang (hairpinA-GCU ${ }_{14}$ ) as a substrate, we found that WT Dis3L2 is distributive for the first step $(P=0.23 \pm 0.003)$, requiring approximately 3 binding events before cleaving the first nucleotide (Fig. 3a-c). This may serve as an important checkpoint before initiation of processive degradation in the second phase, where multiple nucleotides are cleaved before a dissociation event (Fig. 3c).

During the course of the reaction, as the RNA is progressively shortened from the 3 ' end, the $k_{\text {off }}$ decreases (Fig. 3d). We observe a steeper decline in the $k_{\text {off }}$ starting around 11-nt, which could reflect additional stabilizing interactions that become available when the phosphate backbone of the double-helical portion of the substrate is brought into contact with the enzyme (as was observed in the hairpinC- $U_{12}$ and hairpinD- $\mathrm{U}_{7}$ structures). The $k_{\mathrm{on}}$ also decreases with RNA length, likely due to the loss of substrate interaction points (fewer 3'-U binding sites) necessary for stable association (Fig. 3e). Interestingly, the $k_{\mathrm{f}}$ varies with the stage of substrate degradation (Fig. 3d). After an initial slow step, $k_{\mathrm{f}}$ increases as the enzyme degrades through the ss-overhang. When the overhang is shortened to $11 \mathrm{nt}, k_{\mathrm{f}}$ peaks and then begins to fall as the enzyme encounters the dsRNA. This suggests that catalysis and/or translocation slow down as the dsRNA is engaged and later unwound, as $k_{\mathrm{f}}$ reflects the slower of these two processes. Overall, $k_{\text {off }}$ has the dominant effect on processivity across all intermediate species (Fig. 3c). 
Meze et al.

We compared this profile to that of HsDis3, an exosome associated nuclease of this family that, in contrast to Dis3L2, cannot independently degrade structured substrates (Extended data Fig. 8a) ${ }^{4}$. HsDis3

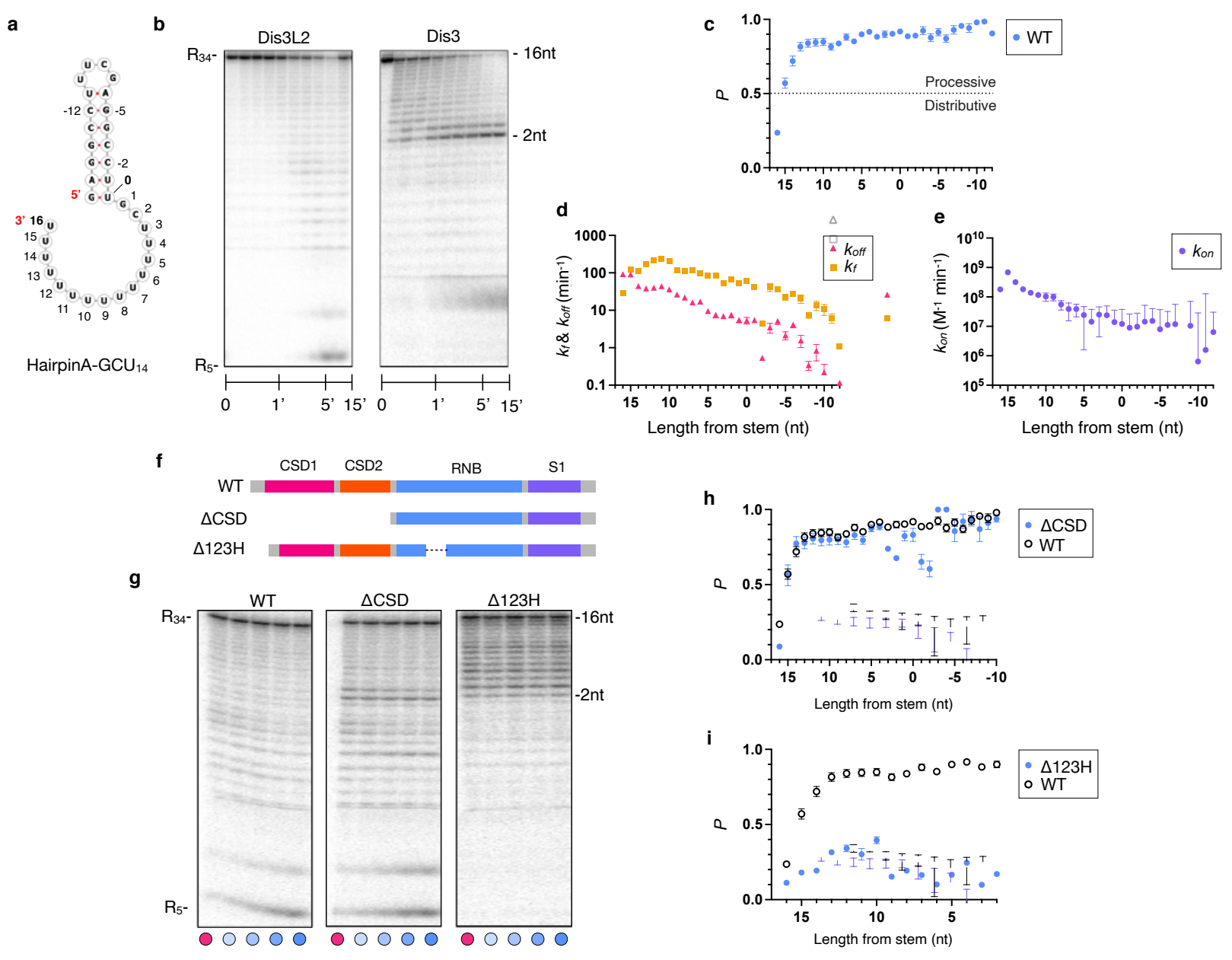

Figure 3. Kinetic profile of structured RNA degradation by WT and mutant HsDis3L2 at single nucleotide resolution. a, Schematic of hairpinA-GCU ${ }_{14}$, for simplicity the kinetic data is numbered from 16 ( 3 ' end) to 0 (ss-ds junction) to denote the nucleotide position. $\mathbf{b}$, Two representative gels from presteady state nuclease titration assays with $1 \mathrm{nM} 5^{\prime} \mathrm{P}^{32}$-radiolabeled hairpinA-GCU ${ }_{14}$ and $25 \mathrm{nM}$ HsDis3L2 and HsDis3, respectively. The overall length of the species and the ss-overhang length are indicated on the left and right of the panels, respectively. c, Processivity $(\mathrm{P}), \mathbf{d}$, dissociation rate constants $\left(\mathrm{k}_{\text {off }}\right)$ and forward rate constants $\left(k_{f}\right), \mathbf{e}$, association rate constants $\left(k_{o n}\right)$ of WT human Dis3L2. $k_{o n}$ datapoint $\mathrm{x}=-8$ was removed due to large uncertainty value. $\mathrm{X}$-axis shows number of nucleotides from the start of the double-stranded stem. f, Domain composition of WT human Dis3L2, $\triangle \mathrm{CSD}$, and $\Delta 123 \mathrm{H}$ deletion mutants. b, Representative gels from pulse-chase reactions of WT human Dis3L2, $\Delta$ CSD, and $\Delta 123 \mathrm{H}$ at $50 \mathrm{nM}$ concentration and $1 \mathrm{nM}$ radiolabeled hairpinA-GCU ${ }_{14}$. Cold chase was added to the reaction at the $3 \mathrm{~min}$ timepoint to a final concentration $5000 \mathrm{nM}$. Timepoints were taken pre-chase at $3 \mathrm{~min}$ (pink dot), and postchase at 4, 5, 7.5, and $10 \mathrm{~min}$ (blue gradient dots). Comparison of Processivity (P) for c, WT and $\triangle \mathrm{CSD}$, and $\mathbf{d}$, WT and $\Delta 123 \mathrm{H}$. Errors for plots $\mathbf{d}$ and $\mathbf{e}$ represent standard errors of the mean (SEM), while processivity plots $\mathbf{c}, \mathbf{h}$, and $\mathbf{i}$ show errors calculated from the SEM of $k_{f}$ and $k_{\text {off }}$ (Methods). 
appears to bind the oligo- $\mathrm{U}$ tailed substrate much tighter and enters directly into processive degradation (Extended data Fig. 8b, c). It maintains high processivity up until the ss-overhang reaches 3 to 2 -nts in length, at which point there is a drastic decrease in processivity as a result of a large increase in the dissociation rate $\left(k_{\text {off }}\right)$ (Fig 3b, Extended data Fig. 8b, e). This shows that unlike HsDis3L2, HsDis3 is not able to maintain sufficient association with the substrate once it encounters the structured portion of the substrate.

\section{The cold shock domains play multiple roles in substrate processing}

Since the CSDs appeared to be the initial recognition sites for the RNA, but then triggered to move to the other side of the protein upon RNA processing, we tested whether they contribute predominantly to initial substrate association or ssRNA degradation. Removal of the CSDs ( $\triangle \mathrm{CSD}$ : A326-S885) led to much lower processivity for the very first step, largely due to a lower forward rate constant, indicating that the CSDs play a role in augmenting the rate of catalysis for the first nt cleavage (Fig. 3f-h, Extended data Fig. 9a). During the following ssRNA degradation steps, $\triangle \mathrm{CSD}$ has a similar processivity to WT Dis3L2 (Fig. 3h). However, there is a significant decrease in the processivity as $\triangle \mathrm{CSD}$ approaches the structured part of the RNA, showing a marked reduction at the 3 and 2 nt ss-overhang position, as well as at -1 and $-2 \mathrm{nt}$ positions, which now fall within the RNA stem. This is a result of a significant increase in the dissociation rate ( $\left.k_{\text {off }}\right)$ (Fig 3h, Extended data Fig. 9b). Thus, the CSDs contribute to both initiation of RNA degradation and maintenance of substrate association during the initial unwinding steps (Supplementary discussion).

\section{The RNB trihelix and linker are necessary for resolving RNA structure}

The Dis3L2-hairpinD-U $\mathrm{U}_{7}$ complex structure shows that the trihelix-linker provides the final barrier before the narrow tunnel to the active site, suggesting a role in dsRNA unwinding. Deletion of the trihelix and linker (residues P612-M669: $\Delta 123 \mathrm{H}$ ) had a striking effect on substrate degradation and a build-up of intermediate species was observed at lengths close to the start of the double strand of hairpinA-GCU (Fig. $^{2}$ $3 \mathrm{f}, \mathrm{g}, \mathrm{i}) . \Delta 123 \mathrm{H}$ never reaches the processive phase, though there is a slight increase in the processivity 
Meze et al.

during initial degradation of the ssRNA overhang. When the substrate shortens to $10 \mathrm{nt}$ in the overhang the dissociation rate $\left(k_{\mathrm{off}}\right)$ increases significantly and the forward rate $\left(k_{\mathrm{f}}\right)$ plateaus leading to a dramatic drop in processivity and a buildup of species with 3, and 2 nt overhangs (Extended data Fig. 9d, e). However, no such build-up was observed in the case of a single strand $\mathrm{U}_{34}$ substrate, demonstrating that the trihelixlinker module is crucial for ds-, but not ss-RNA degradation (Extended data Fig. 9g-j).

\section{A comprehensive model for structured RNA degradation}

Combining our cryoEM and kinetic data, we propose the following model: RNA degradation by Dis3L2 proceeds via a minimum of six sequential steps: 1) substrate association and quality control, 2) initial nucleotide cleavage, 3) 3' single-strand degradation, 4) double-strand engagement, 5) dramatic domain realignment, and 6) concurrent double-strand unwinding and degradation (Fig. 4). During the first four stages, Dis3L2 is in the vase conformation, with the S1 and CSDs positioned to form a large, positivelycharged surface for the oligo-U tail of the RNA (Fig 1b, Extended data Fig. 1f). While the S1 and RNB domains provide crucial binding interactions, the CSDs enable the effective initiation of degradation by contributing to the first catalytic step (Fig. 3h, Extended data Fig. 9a). This initial step is slow, and acts as a substrate checkpoint. Once cleared, the enzyme enters the highly processive phase (Fig. 3c). When the overhang is shortened to 11-12 nt, the RNA duplex engages with the enzyme, stabilized by contacts with the S1 and CSDs (Fig. 1d). At this point, the forward rate constant begins to decrease as the base of the dsRNA hairpin gets closer to the tunnel in the RNB (Fig. 3d). When the ss- 3' overhang reaches 9-8 nt, the 5' strand of the RNA double helix runs into CSD1 and triggers the large movement of the two CSDs to the other side of the enzyme (Fig. 2a-c, SI Movie 1). In the resulting prong conformation, the S1 angles towards the tunnel and engages the backbone of the RNA double helix which now sits over the RNB trihelix-linker (Extended data Fig. 4g). The trihelix-linker module acts as a wedge between the two RNA strands to separate them and enable the 3' strand to enter into the narrow part of the now shortened tunnel (Extended data Fig. 5a). Strand unwinding likely initiates when the overhang reaches roughly $5 \mathrm{nt}$ (Supplementary discussion). Alignment of the structure of Dis3L2 in complex with hairpinD- $\mathrm{U}_{7}$ with known structures of 
Meze et al.

RNase R/II family nucleases suggest that most would have to undergo a similar conformational change in order to allow the ds- portion of the RNA access to the trihelix-linker wedge. Biochemical studies of E. coli RNase R have also demonstrated the importance of the trihelix in dsRNA degradation ${ }^{30}$, suggesting that the mechanism proposed here could be conserved in other members of the RNase R/II family of nucleases (Supplementary discussion). Collectively, this work unveils a new molecular mechanism for efficient, regulatory degradation of structured RNAs by a vital nuclease.

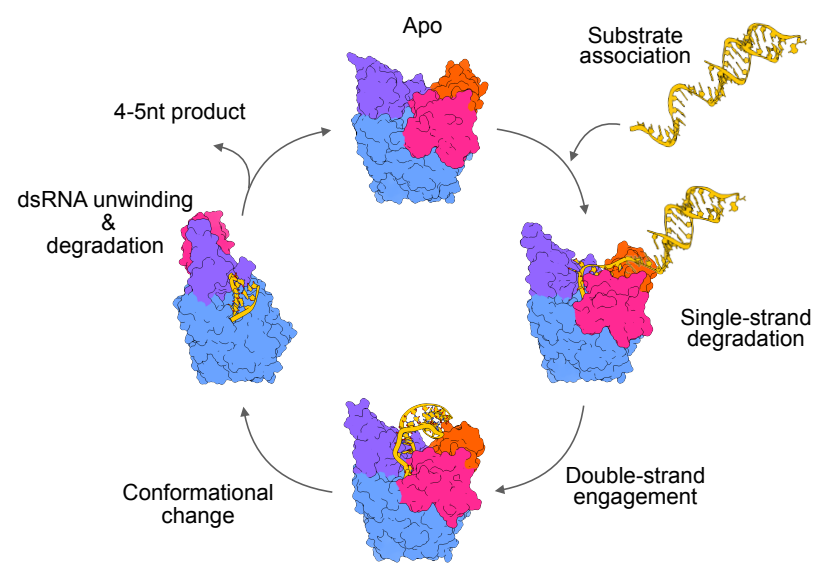

Figure 4. Model of structured RNA processing by Dis3L2. RNA-free Dis3L2 is pre-organized into a vase conformation to bind RNA substrates (yellow), with a 7nt deep tunnel leading to the nuclease active site. When the RNA overhang is shortened to $\sim 12$ nt, additional contacts are made to the dsRNA. Further shortening of the overhang triggers a large rearrangement of the two CSD domains (pink, orange) to the prong conformation, and allows the base of the dsRNA to access a module in the RNB domain (blue) that acts as a wedge to separate to two RNA strands and allow entry of one of the strands into the narrow tunnel leading to the active site. In this way the enzyme ensures continued RNA degradation during RNA-duplex unwinding. 


\section{References}

1. Astuti, D. et al. Germline mutations in DIS3L2 cause the Perlman syndrome of overgrowth and Wilms tumor susceptibility. Nat. Genet. 44, 277-284 (2012).

2. Chang, H.-M., Triboulet, R., Thornton, J. E. \& Gregory, R. I. A role for the Perlman syndrome exonuclease Dis312 in the Lin28-let-7 pathway. Nature 497, 244-8 (2013).

3. Malecki, M. et al. The exoribonuclease Dis3L2 defines a novel eukaryotic RNA degradation pathway. EMBO J. 32, 1842-1854 (2013).

4. Lubas, M. et al. Exonuclease hDIS3L2 specifies an exosome-independent $3^{\prime}-5^{\prime}$ degradation pathway of human cytoplasmic mRNA. EMBO J. 32, 1855-1868 (2013).

5. Ustianenko, D. et al. Mammalian DIS3L2 exoribonuclease targets the uridylated precursors of let-7 miRNAs. Rna 19, 1632-1638 (2013).

6. Faehnle, C. R., Walleshauser, J. \& Joshua-Tor, L. Mechanism of Dis312 substrate recognition in the Lin28-let-7 pathway. Nature 514, 252-256 (2014).

7. Nowak, J. S. et al. Lin28a uses distinct mechanisms of binding to RNA and affects miRNA levels positively and negatively. RNA 23, 317-332 (2017).

8. Xing, S. et al. DIS3L2 Promotes Progression of Hepatocellular Carcinoma via hnRNP UMediated Alternative Splicing. Cancer Res. 79, 4923-4936 (2019).

9. Towler, B. P., Jones, C. I., Harper, K. L., Waldron, J. A. \& Newbury, S. F. A novel role for the $3^{\prime}-5$ ' exoribonuclease Dis3L2 in controlling cell proliferation and tissue growth. $R N A$ Biol. 13, 1286-1299 (2016).

10. Hunter, R. W. et al. Loss of Dis312 partially phenocopies perlman syndrome in mice and results in upregulation of Igf2 in nephron progenitor cells. Genes Dev. 32, 903-908 (2018).

11. Towler, B. P. et al. Dis3L2 regulates cell proliferation and tissue growth though a conserved mechanism. PLoS Genet. 16, 1-29 (2020).

12. Pirouz, M. et al. The Perlman syndrome DIS3L2 exoribonuclease safeguards endoplasmic reticulum-targeted mRNA translation and calcium ion homeostasis. Nat. Commun. 11, 113 (2020).

13. Thomas, M. P. et al. Apoptosis Triggers Specific, Rapid, and Global mRNA Decay with 3' Uridylated Intermediates Degraded by DIS3L2. Cell Rep. 11, 1079-1089 (2015).

14. Liu, X. et al. PNPT1 Release from Mitochondria during Apoptosis Triggers Decay of Poly(A) RNAs. Cell 174, 187-201.e12 (2018).

15. Lim, J. et al. Uridylation by TUT4 and TUT7 marks mRNA for degradation. Cell 159, 13651376 (2014).

16. Yang, A. et al. AGO-bound mature miRNAs are oligouridylated by TUTs and subsequently degraded by DIS3L2. Nat. Commun. 11, 1-13 (2020).

17. Ustianenko, D. et al. TUT-DIS3L2 is a mammalian surveillance pathway for aberrant structured non-coding RNAs. EMBO J. 35, 2179-2191 (2016).

18. Sun Lee, Y. \& Dutta, A. The tumor suppressor microRNA let-7 represses the HMGA2 
oncogene. Genes Dev. 21, 1025-1030 (2007).

19. Mayr, C., Hemann, M. T. \& Bartel, D. P. Disrupting the Pairing Between let-7 and Hmga2 Enhances Oncogenic Transformation. Science (80-. ). 315, 1576-1579 (2007).

20. Yu, F. et al. let-7 Regulates Self Renewal and Tumorigenicity of Breast Cancer Cells. Cell 131, 1109-1123 (2007).

21. Kumar, M. S., Lu, J., Mercer, K. L., Golub, T. R. \& Jacks, T. Impaired microRNA processing enhances cellular transformation and tumorigenesis. Nat. Genet. 39, 673-677 (2007).

22. Johnson, S. M. et al. RAS is regulated by the let-7 microRNA family. Cell 120, 635-647 (2005).

23. Viswanathan, S. R. et al. Lin28 promotes transformation and is associated with advanced human malignancies. Nat. Genet. 41, 843-848 (2009).

24. Shukla, S., Bjerke, G. A., Muhlrad, D., Yi, R. \& Parker, R. The RNase PARN Controls the Levels of Specific miRNAs that Contribute to p53 Regulation. Mol. Cell 73, 1204-1216.e4 (2019).

25. Haas, G. et al. Identification of factors involved in target RNA-directed microRNA degradation. Nucleic Acids Res. 44, 2873-2887 (2016).

26. Roithová, A., Feketová, Z., Vaňáčová, Š. \& Staněk, D. DIS3L2 and LSm proteins are involved in the surveillance of Sm ring-deficient snRNAs. Nucleic Acids Res. 48, 61846197 (2020).

27. Pirouz, M., Munafò, M., Ebrahimi, A. G., Choe, J. \& Gregory, R. I. Exonuclease requirements for mammalian ribosomal RNA biogenesis and surveillance. Nat. Struct. Mol. Biol. 26, 490-500 (2019).

28. Pirouz, M., Du, P., Munafo, M. \& Gregory, R. I. Dis312-Mediated Decay Is a Quality Control Pathway for Noncoding RNAs. 1861-1873 (2016). doi:10.1016/j.celrep.2016.07.025

29. Matos, R. G., Barbas, A. \& Arraiano, C. M. RNase R mutants elucidate the catalysis of structured RNA: RNA-binding domains select the RNAs targeted for degradation. Biochem. J. 423, 291-301 (2009).

30. Chu, L. Y. et al. Structural insights into RNA unwinding and degradation by RNase R. Nucleic Acids Res. 45, 12015-12024 (2017). 
Meze et al.

\section{Methods}

\section{Protein preparation}

Full-length Human Dis3L2, domain deletions, point mutants, and HsDis3 were cloned as Nterminal Strep-Sumo-TEV (SST) fusion proteins in a pFL vector of the MultiBac Baculovirus expression system ${ }^{31}$. Benchling (https://www.benchling.com) was used for sequence analysis and primer design. Expression and purification followed a similar protocol as detailed in Faehnle et al 20146. All constructs were expressed in SF9 cells grown in Hyclone CCM3 media (Thermo Scientific) at $27^{\circ} \mathrm{C}$ for $60 \mathrm{~h}$. Cells were then pelleted and resuspended in wash buffer ( $50 \mathrm{mM}$ Tris pH8, $100 \mathrm{mM} \mathrm{NaCl}, 5 \mathrm{mM}$ DTT) and a protease inhibitor cocktail added before snap freezing with liquid $\mathrm{N}_{2}$ for storage at $-80^{\circ} \mathrm{C}$. After thawing, cells were lysed by increasing $\mathrm{NaCl}$ to $500 \mathrm{mM}$ followed by one round of sonication. $0.1 \%$ poly-ethylene imine (PEI) was added to the lysate and cell debris cleared by 45 min of ultracentrifugation at 35,000 r.p.m. at $4^{\circ} \mathrm{C}$. The solution was then incubated with Strep-Tactin superflow resin (IBA bioTAGnology) for 30 minutes while on a rolling shaker. The slurry was applied to a gravity column and washed with 20 column volumes of wash buffer before eluting the protein with $2 \mathrm{mM}$ desthiobiotin in wash buffer. The SST tag was cleaved using TEV protease overnight at $4^{\circ} \mathrm{C}$. Cleavage efficiency and sample purity were assessed by SDS-PAGE. The protein was then diluted to a final salt concentration of $50 \mathrm{mM}$ and $25 \mathrm{mM}$ HEPES pH 7.5, $5 \mathrm{mM}$ DTT, and applied to a HiTrap heparin HP affinity purification column (GE Life Sciences) equilibrated in $50 \mathrm{mM} \mathrm{NaCl}, 25 \mathrm{mM}$ HEPES pH 7.5, $5 \mathrm{mM}$ DTT. The bound protein was eluted by applying a linear increasing salt gradient $(0.05 \mathrm{M}$ to $1 \mathrm{M} \mathrm{NaCl})$. Pooled fractions of protein were then concentrated and loaded onto a 10/300 Superdex200 Increase gel filtration column (GE Life Sciences) equilibrated in $20 \mathrm{mM}$ Hepes pH 7.5, $150 \mathrm{mM} \mathrm{NaCl}$ and 
Meze et al.

5 mM DTT. Protein purity was assessed by the quality of the chromatogram and by running SDS-

PAGE gels. The concentrated sample was frozen in liquid $\mathrm{N}_{2}$ and stored at $-80{ }^{\circ} \mathrm{C}$.

\section{Cryo-EM sample and grid preparation}

RNA oligos were purchased from Dharmacon and RNA secondary structure predictions were done using the Vienna RNAfold web server (http://rna.tbi.univie.ac.at) and diagrams made using Forna (Fig 3a, Extended Data Fig 2a, 3a) $)^{32-34}$. RNA hairpins were annealed by diluting into $20 \mathrm{mM}$ Hepes $\mathrm{pH} 7.5,150 \mathrm{mM} \mathrm{NaCl}$ and $5 \mathrm{mM}$ DTT and heated to $95^{\circ} \mathrm{C}$ for 3 minutes before stepwise cooling $\left(95{ }^{\circ} \mathrm{C}, 50{ }^{\circ} \mathrm{C}, 30^{\circ} \mathrm{C}, 4^{\circ} \mathrm{C}\right)$. Complexes of human Dis3L2 and various RNAs were prepared by mixing equimolar ratios of Dis3L2 and RNA, incubating for 15 min and loading onto a 10/300 Superdex200 Increase gel filtration column (GE Life Sciences) equilibrated in the same buffer (for specific controls indicated in Extended data Fig. 6b $100 \mu \mathrm{M}$ EDTA was added to the buffer). Complex formation was evaluated by monitoring a peak shift and the ratio of absorbance at 260 and $280 \mathrm{~nm}$. Fractions of the complex were then pooled and concentrated to roughly 0.5 $\mathrm{mg} / \mathrm{ml}$ for Quantifoil carbon-coated $\mathrm{Cu}$ grids, or $0.3 \mathrm{mg} / \mathrm{ml}$ for Au-foil grids (Quantifoil, Jena, DE). 4 ul of sample was applied to glow-discharged grids, and a Vitrobot plunger (Thermo Fisher Scientific) was used to freeze the grids in liquid ethane (humidity $95 \%, 20{ }^{\circ} \mathrm{C}$, blot force 4 , blot time 2.5s).

\section{Cryo-EM data acquisition and image processing}

Data were collected on a $300 \mathrm{kV}$ Titan Krios electron microscope at either 215,000X (0.84 pixel size) or $130,000 \mathrm{X}(0.64 \AA$ pixel size $)$ on a Gatan K2 or K3 detector equipped with an energy filter. A similar pipeline was used for all datasets (see below). CTF estimation, motion correction 
Meze et al.

and particle picking were done concurrent to data collection with Warp-EM ${ }^{35}$. Good particles (as selected by WarpEM) were imported into CryoSPARC where 2D classification was done. A subselection of particles was made taking the best 2D classes with the highest resolution (below $4 \AA$ for those that led to a high-resolution structure) and largest particle number (classes with more than 5000 particles) with protein-like features. This particle selection was then used in multi-class $a b$ initio reconstruction. The classes were evaluated and then used as starting references for heterogeneous refinement, this time using all the good particles from Warp's picking process. The best heterogeneous refinement classes and their particle subsets were then used for homogeneous refinement and in some cases non-uniform refinement in cryoSPARC (Structura Biotechnology) $)^{36,37}$. The Dis3L2-hairpinA-GCU ${ }_{14}$ dataset was also processed in Relion using their 3D classification, refinement, CTF refinement and particle polishing ${ }^{38-40}$. Two representative

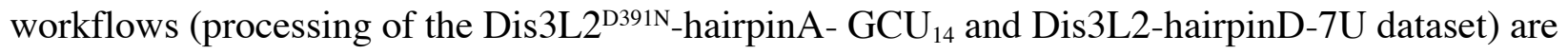
shown in SI Fig. 1.

In order to assess the distribution of particles between different Dis3L2 conformations we used the following standardized protocol for data processing: 1) particles from the datasets were picked using Warp-EM's neural network-based picker, 2) good particles were then classified in CryoSPARC's 2D classification, 3) the best 2D classes (as described above) were selected for $a b$ initio reconstruction using 5 classes, and 4) the resulting 5 ab initio models were used as starting references for heterogeneous refinement using all of the good particles found by the Warp-EM picker (Extended data Fig. 5a). This allowed us to compare the proportion of particles in the full dataset that contributed to a particular Dis3L2 conformation. To ensure that the class distributions were not a result of RNA degradation, control datasets with EDTA were also analyzed and showed the same overall distribution (Extended data Fig. 5b). 
Meze et al.

\section{Model building and refinement}

Model building and refinement were done in Coot and Phenix ${ }^{41-43}$. Since the mouse and human Dis3L2 proteins are extremely similar in sequence, the mouse Dis3L2 structure (pdb: 4pmw) was used as a starting reference for model building 6 . Once the reference structure was fit into the cryoEM map, Real-Space Refine (RSR) was used, using morphing, simulated annealing and rigid body fit in the first rounds ${ }^{42}$. After manual building and correction of geometric outliers and clashes using Coot, further rounds of refinement were done using both secondary structure restrictions as well as global minimization, refinement of atomic displacement parameters (ADP or B-factors) and local grid search. Refinements of complexes with RNA contained further base-pair and basestacking restraints in the double-stranded regions. RNA-protein interactions were found with PDBePISA (https://www.ebi.ac.uk/pdbe/pisa/) and examined manually. Final model validation metrics are provided in Supplementary Information Table 1. Electrostatics were calculated using PyMol 2.2.3 (Schrodinger, LLC) at an ionic strength of $150 \mathrm{mM}$.

\section{Pre-steady-state and quasi-steady-state nuclease reactions}

Nuclease reactions were performed in a temperature-controlled heat block at $20^{\circ} \mathrm{C}$ in a total volume of $40 \mu \mathrm{L}$. Reaction mixtures containing $20 \mathrm{mM}$ HEPES, $\mathrm{pH} 7.0,50 \mathrm{mM} \mathrm{NaCl}, 5 \%$ glycerol, $100 \mu \mathrm{M} \mathrm{MgCl}_{2}, 1 \mathrm{mM}$ DTT and Dis3L2 were pre-incubated for 5 minutes. Pre-steady state reactions were started by the addition of 5'-radiolabeled RNA substrate to a final concentration of $1 \mathrm{nM}$. The concentrations of Dis3L2 were in far excess of the RNA and ranged from $5 \mathrm{nM}$ to $1000 \mathrm{nM}$, as indicated. Timepoints were taken at $7 \mathrm{~s}, 15 \mathrm{~s}, 30 \mathrm{~s}, 1^{\prime}, 2^{\prime}, 3^{\prime}, 5^{\prime}, 10^{\prime}$ and $15^{\prime}$, except for long time-course experiments where the times are indicated (Extended data Fig. $7 b, c)$. Reactions were quenched by the addition to an equal volume of stop buffer ( $80 \%$ 
formamide, $0.1 \%$ bromophenol blue, $0.1 \%$ xylene cyanole, 2 mM EDTA and 1.5 M urea). Samples were heated to $95{ }^{\circ} \mathrm{C}$ and analyzed on sequencing gels composed of $20 \%$ acrylamide and $7 \mathrm{M}$ urea. Gels were exposed to phosphor screens overnight and scanned with a Typhoon FLA 7000 (GE Healthcare Life Sciences) imager. Bands were quantified using SAFA footprinting software and the values normalized for each lane ${ }^{44}$. For a typical reaction with a 34 nt substrate and 10 timepoints, we quantified all species larger than the $5 \mathrm{nt}$ end-product and obtained approximately 300 datapoints for each Dis3L2 concentration.

\section{Pulse-chase nuclease reactions}

Pulse-chase reactions were performed under conditions identical to those for pre-steady state reactions. Reactions were initiated by the addition of enzyme and allowed to proceed for a defined period of time $\left(t_{1}\right)$. At $t_{1}$, an excess of cold scavenger RNA (x 5000-fold) was added to a final concentration of $5 \mu \mathrm{M}$. After incubation for the indicated time $\left(\mathrm{t}_{2}\right)$, aliquots were removed and quenched in stop buffer. Samples were analyzed on sequencing gels and processed as the abovedescribed pre-steady state reactions.

\section{Calculation of kinetic parameters}

Kinetic parameters were obtained using a global fit of the data from pre-steady state titrations and pulse-chase experiments. Global data fitting was performed using the Kinetic Explorer software, version 8.0 (Kintek Global) ${ }^{45,46}$. Initial parameters for the global fit were: observed rate constants $\left(k_{\mathrm{obs}}\right)$, processivity values $(P), K_{1 / 2}$ and $k_{\mathrm{obs}}{ }^{\max }$ values for each reaction species. Observed rate constants $\left(k_{\mathrm{obs}}\right)$ were calculated from pre-steady state experiments by fitting each experiment separately using the global data-fitting software GFIT $^{47}$ to a model that calculates rate constants 
for a series of irreversible, pseudo-first-order reactions. Initial parameters for GFIT were obtained by fitting the disappearance of $34 \mathrm{nt}$ substrate to a first order exponential: $\mathrm{y}=\mathrm{a} 1 * \exp (-\mathrm{b} 1 * \mathrm{t})+\mathrm{c}$, where a1 is the amplitude, b1 is the observed rate constant $\left(k_{o b s}\right)$ and $\mathrm{c}$ is the offset. Processivity values for individual degradation steps were determined from the distribution of substrate species before and after scavenger addition ${ }^{48}($ Extended data Fig. $7 \mathrm{~g})$, where Processivity $(P)$ is defined as: $P=k_{\mathrm{f}} /\left(k_{\mathrm{f}}+k_{\mathrm{off}}\right)$.

The equations to calculate processivity values from distributions of species are fit using a customized script using the Mathematica software package (Wolfram) ${ }^{48}$. To derive the $K_{1 / 2}$ and $k_{\mathrm{obs}}{ }^{\max }$ values, we fit $k_{\mathrm{obs}} v s$. Dis3L2 concentration data to a binding isotherm function defined as: $k_{\mathrm{obs}}=\left(k_{\mathrm{obs}}{ }^{\max } \cdot[\mathrm{Dis} 3 \mathrm{~L} 2]\right) \cdot\left(K_{1 / 2}{ }^{\mathrm{Dis} 3 \mathrm{~L} 2}+[\mathrm{Dis} 3 \mathrm{~L} 2]\right)^{-1} \cdot K_{1 / 2}$ is the functional equilibrium dissociation constant and $k_{\mathrm{obs}}{ }^{\max }$ is the maximal observed rate constant at enzyme saturation (Extended data Fig. 7d). The data were then evaluated by plotting in GraphPad Prism version 9.1.2. (GraphPad Software). These initial parameters were used as guides in setting up a range of starting values for the elementary rate constants in a global fit to the minimal kinetic model as shown in Extended Data Fig. 7a. The $K_{1 / 2}$ values were used to constrain the ratio of dissociation and association rate constants for productive binding by linking the two values as initial parameters. The $k_{o b s}{ }^{\max }$ values were used to set boundaries on the forward rate constant, $k_{f}$. Finally, the experimentally determined processivity values $(P)$ were used as initial constraints on the ratio of $k_{f}$ and $k_{\text {off }}$. The global data fit was done in an iterative manner by alternating combinations of fixed and floating variables while tracking the overall $\chi^{2}$ value. The goodness of the fit, $\mathrm{R}^{2}$ was 0.94 , calculated by plotting the experimental datasets $v s$ the corresponding simulated data from the kinetic model (Extended data Fig. 7f). As an additional measure of the overall quality of fit we performed Fitspace analysis ${ }^{46}$ to determine the lower and upper boundaries of each kinetic parameter (Supplementary Information 
Meze et al.

Table 2-4). For a typical substrate, roughly 2500 individual datapoints from enzyme titrations and 750 datapoints from pulse-chase experiments were used to calculate the 120 kinetic parameters that describe degradation of a 34 nt substrate down to $5 \mathrm{nt}$.

Errors for elementary rate constants represent standard errors of the mean from the global data fitting. Errors for compound rate constants such as processivity and $\mathrm{K}_{1 / 2}$ were calculated via error propagation formulas shown below.

Equation 1. Error propagation formula for $K_{1 / 2}$ and Processivity.

$$
\begin{gathered}
\sigma_{K_{1 / 2}}=K_{1 / 2} \sqrt{\frac{\sigma_{k_{\text {off }}}^{2}}{k_{\text {off }}^{2}}+\frac{\sigma_{k_{\text {on }}}^{2}}{k_{\text {on }}^{2}}} \\
\sigma_{P}=P(1-P) \sqrt{\frac{\sigma_{k_{\text {off }}}^{2}}{k_{\text {off }}^{2}}+\frac{\sigma_{k_{\mathrm{f}}}^{2}}{k_{\mathrm{f}}^{2}}}
\end{gathered}
$$

\section{Data availability}

CryoEM data and structure coordinates will be deposited prior to publication in the EM-DB and PDB respectively. 


\section{Methods and supplementary discussion references}

31. Bieniossek, C., Richmond, T. J. \& Berger, I. MultiBac: Multigene baculovirus-based eukaryotic protein complex production. Curr. Protoc. Protein Sci. 1-26 (2008). doi:10.1002/0471140864.ps0520s51

32. Kerpedjiev, P., Hammer, S. \& Hofacker, I. L. Forna (force-directed RNA): Simple and effective online RNA secondary structure diagrams. Bioinformatics 31, 3377-3379 (2015).

33. Gruber, A. R., Lorenz, R., Bernhart, S. H., Neuböck, R.\& Hofacker, I. L. The Vienna RNA websuite. Nucleic Acids Res. 36, 70-74 (2008).

34. Mathews, D. H. et al. Incorporating chemical modification constraints into a dynamic programming algorithm for prediction of RNA secondary structure. Proc. Natl. Acad.Sci. U. S. A. 101, 7287-7292 (2004).

35. Tegunov, D. \& Cramer, P. Real-time cryo-EM data pre-processing with Warp. (2018).

36. Punjani, A., Rubinstein, J. L., Fleet, D. J. \& Brubaker, M. A. CryoSPARC: Algorithms for rapid unsupervised cryo-EM structure determination. Nat. Methods 14, 290-296 (2017).

37. Punjani, A., Zhang, H. \& Fleet, D. J. Non-uniform refinement: adaptive regularization improves single-particle cryo-EM reconstruction. Nat. Methods 17, 1214-1221 (2020).

38. Zivanov, J. et al. New tools for automated high-resolution cryo-EM structure determination in RELION-3. Elife 7, 1-22 (2018).

39. Scheres, S. H. W. Processing of Structurally Heterogeneous Cryo-EM Data in RELION. Methods in Enzymology 579, (Elsevier Inc., 2016).

40. Scheres, S. H. W. RELION: Implementation of a Bayesian approach to cryo-EM structure determination. J. Struct. Biol. 180, 519-530 (2012).

41. Emsley, P. \& Cowtan, K. Coot: Model-building tools for molecular graphics. Acta Crystallogr. Sect. D Biol. Crystallogr. 60, 2126-2132 (2004).

42. Afonine, P. V. et al. Real-space refinement in PHENIX for cryo-EM and crystallography. Acta Crystallogr. Sect. D Struct. Biol. 74, 531-544 (2018).

43. Terwilliger, T. C., Ludtke, S. J., Read, R. J., Adams, P. D. \& Afonine, P. V. Improvement of cryo-EM maps by density modification. Nat. Methods 17, 923-927 (2020).

44. Das, R., Laederach, A., Pearlman, S. M., Herschlag, D. \& Altman, R. B. SAFA: Semiautomated footprinting analysis software for high-throughput quantification of nucleic acid footprinting experiments. Rna 11, 344-354 (2005).

45. Johnson, K. A., Simpson, Z. B. \& Blom, T. Global Kinetic Explorer: A new computer program for dynamic simulation and fitting of kinetic data. Anal. Biochem. 387, 20-29 (2009).

46. Johnson, K. A., Simpson, Z. B. \& Blom, T. FitSpace Explorer: An algorithm to evaluate multidimensional parameter space in fitting kinetic data. Anal. Biochem. 387, 30-41 (2009).

47. Levin, M. K., Hingorani, M. M., Holmes, R. M., Patel, S. S. \& Carson, J. H. Model-Based Global Analysis of Heterogeneous Experimental Data Using gfit. 500, 494 (2009). 
48. Axhemi, A., Wasmuth, E. V, Lima, C. D. \& Jankowsky, E. Substrate selectivity by the exonuclease Rrp6p. PNAS 117, 982-992 (2019).

49. Bayne, R. A. et al. Yeast Ssd1 is a non-enzymatic member of the RNase II family with an alternative RNA recognition interface. bioRxiv 1-30 (2020). doi:10.1101/2020.10.22.350314

50. Makino, D. L. et al. RNA degradation paths in a 12-subunit nuclear exosome complex. Nature 524, 54-58 (2015).

51. Bian, Y. et al. An enzyme assisted RP-RPLC approach for in-depth analysis of human liver phosphoproteome. J. Proteomics 96, 253-262 (2014).

52. Ballou, E. R., Cook, A. G. \& Wallace, E. W. J. Repeated Evolution of Inactive Pseudonucleases in a Fungal Branch of the Dis3/RNase II Family of Nucleases. Mol. Biol. Evol. 1-10 (2020). doi:10.1093/molbev/msaa324

53. Jansen, J. M., Wanless, A. G., Seidel, C. W. \& Weiss, E. L. Cbk1 Regulation of the RNABinding Protein Ssd1 Integrates Cell Fate with Translational Control. Curr. Biol. 19, 2114-2120 (2009).

54. Weick, E. M. et al. Helicase-Dependent RNA Decay Illuminated by a Cryo-EM Structure of a Human Nuclear RNA Exosome-MTR4 Complex. Cell 173, 1663-1677.e21 (2018).

55. Gerlach, P. et al. Distinct and evolutionary conserved structural features of the human nuclear exosome complex. Elife 1-22 (2018). doi:10.7554/eLife.38686.001

56. Lee, G., Bratkowski, M. A., Ding, F., Ke, A. \& Ha, T. Elastic coupling between RNA degradation and unwinding by an exoribonuclease. Science (80-. ). 336, 1726-1729 (2012).

57. Lv, H. et al. Structural analysis of Dis312, an exosome-independent exonuclease from Schizosaccharomyces pombe. Acta Crystallogr. Sect. D Biol. Crystallogr. 71, 1284-1294 (2015). 
Meze et al.

\section{Acknowledgements}

We thank Daniel Herschlag for detailed discussions, comments and recommendations, Justin Kinney for advice, Seraya Jones and John Scheuring for technical support, Jonathan Ipsaro for comments on the manuscript, Matt Jaremko for technical advice and other members of the JoshuaTor lab for helpful suggestions. Cryo-EM was performed at the CSHL cryo-EM facility. We also thank the CSHL Mass Spectrometry Shared Resource, which is supported by the Cancer Center Support Grant 5P30CA045508. This work was supported by NIH grant R01-GM114147 (to L.J.), the CSHL School of Biological Sciences (to K.M., A.D. and L.J.). K.M. was supported by the Leslie C. Quick, Jr. Fellowship. L.J. is an Investigator of the Howard Hughes Medical Institute.

\section{Competing interest declaration}

The authors have no competing interest

\section{Contributions}

K.M. and L.J. designed the study. K.M. purified the proteins used in this work, and preformed all cryoEM sample preparation, data collection, data processing and model building. D.R.T. assisted in cryoEM data collection and A.D. contributed to the purification and cryoEM analysis of RNA-free and hairpinA-GCU ${ }_{14}$ HsDis3L2. A.A. designed and lead the kinetic analysis of HsDis3L2. A.A and K.M. carried out the kinetic assays and data analysis. K.M., A.A. and L.J prepared the manuscript.

\section{Additional information}

Additional Supplementary Information and Movie are provided in separate files. 